HL in all studies was $22.7 \%$ (95\% CI 20.6\%, 24.8\%). The review identified associations between limited HL and socioeconomic factors (lower education attainment, lower income), and certain process and outcome measures (lower likelihood of referral for transplant, higher mortality). Overall study quality was poor, with particular weaknesses of sampling and non-response.

Conclusion Limited health literacy is common among people with $\mathrm{CKD}$ and independently associated with socioeconomic factors and health outcomes. It may represent an important determinant of inequality of outcomes in CKD. There is a need for further investigation of limited health literacy in people with pre-end stage CKD, and in UK CKD populations.

\section{PS27 SERUM BILIRUBIN AND RISK OF CARDIOVASCULAR EVENTS AND DEATH IN A STATIN-TREATED POPULATION: A COHORT STUDY}

doi:10.1136/jech-2012-201753.126

LJ Horsfall, I Petersen, I Nazareth. Primary Care and Population Health, UCL, London, UK

Background Serum bilirubin is an endogenous antioxidant with a strong genetic component that may be a marker for future cardiovascular disease (CVD) risk. We examined the relationship between serum bilirubin levels recorded prior to statin prescription, primarily as test for liver function, and the diagnosis or death from CVD. We also examined whether bilirubin levels increased following statin prescription independently of liver enzymes, which has been shown in animal models and could contribute to the purported health benefits of statins in addition to cholesterol reduction.

Methods All patients with liver function tests three months prior to first statin treatment between January 1st 2000 and December 31 st 2010 and no history of liver disease or CVD were extracted from The Health Improvement Network (THIN) primary care database. Restricted cubic spline Poisson regressions were fitted on bilirubin levels and adjusted for traditional cardiovascular risk factors to estimate incidence rate ratios.

Results In total 130,052 patients met the inclusion criteria and after a median follow-up of 43 months, there were 5,938 coronary heart disease (CHD) events, 2,438 stroke events, and 5,185 deaths from any cause. In men, the incidence of CHD in the lowest decile category of bilirubin $(1-6 \mu \mathrm{mol} / \mathrm{L})$ was 176 per 10,000 person years (PYs) compared with 139 per 10,000 PYs in the highest decile (19-40 $\mu \mathrm{mol} / \mathrm{L})$. The result for stroke was 72 versus 52 per 10,000 PYs and for death 139 versus 92 per 10,000 PYs. Similar differences were seen for women. The adjusted associations with bilirubin were L-shaped with a negative relationship up to around $10-15 \mu \mathrm{mol} / \mathrm{L}$. The models predicted that, compared to patients with the median bilirubin level $(10 \mu \mathrm{mol} / \mathrm{L})$, those with a similar CVD risk profile but a bilirubin level of $5 \mu \mathrm{mol} / \mathrm{L}$ had a $19 \%$ (95\% CI: $11-27 \%$ ) higher rates of CHD, a $22 \%$ (95\% CI: $10-36 \%$ ) higher rates of stroke, and a $26 \%$ (95\% CI: $17-35 \%$ ) higher rate of death. A dose-dependent increase in mean bilirubin level was seen following atorvastatin prescription but not for simvastatin.

Conclusion Low bilirubin prior to statin prescription is an independent risk factor for CVD and death. Further work is needed to examine whether pleotrpic effects of statins can be explained by alterations in bilirubin production and/or elimination.

\section{PS28 IMPACT OF NUMBER AND TYPE OF COMORBIDITY ON DEPRESSION PREVALENCE AND HEALTH CARE COSTS. POPULATION-BASED COHORT STUDY}

doi:10.1136/jech-2012-201753.127

${ }^{1} \mathrm{~N}$ Bhattarai, ${ }^{1} \mathrm{~J}$ Charlton, ${ }^{2} \mathrm{C}$ Rudisill, 'M C Gulliford. 'Department of Primary Care and Public Health Sciences, King's College London, London, UK; ${ }^{2}$ Department of Social Policy and LSE Health, London School of Economics, London, UK
Background The degree to which the number of comorbidities and the presence of depression impact health care use and costs has been unexplored employing actual resource use data representing a general population. We aimed to evaluate the impact of number and type of comorbidities on depression prevalence, health care utilisation and health care costs.

Methods Cohort study and included in the study were 300,020 participants aged 30 years of age registered with the UK General Practice Research Database. We used the UK General Practice Research Database to examine participants' diagnoses and resource use in primary and secondary care from 2005 to 2009. Healthcare unit costs and the costs of each individual prescription item were analysed.

Results In participants with no comorbidity, the age-standardised prevalence of depression was $7 \%$ in men and $14 \%$ in women. The likelihood of having depression increased in participants with single comorbidities including diabetes mellitus (men 13\%, women $22 \%$ ), CHD (men $15 \%$, women $24 \%$ ), stroke (men $14 \%$, women $26 \%$ ) or colorectal cancer (men 10\%, women 21\%). Patients with concurrent diabetes, CHD and stroke had a very high prevalence of depression (men 23\%, women 49\%) with women being more likely to suffer depression. Patients with a single comorbidity were 1.63 (95\% confidence interval 1.59 to 1.66 ) times more likely to be depressed than those without comorbidity while those with two and three comorbidities were 1.96 (1.89 to 2.03) and 2.35 (2.03 to 2.59) times more likely. Depression increased the total costs of resources utilised per year in all participants across both genders, all age groups and across all comorbidities analysed in this study. Individuals with depression had higher total annual health care costs (males $=£ 1014$, females=£1212) than those without comorbidity or depression (males $=£ 380$, females $=£ 517$ ). Those with diabetes alone had $£ 1144$ for males and $£ 1393$ for females but $£ 2534$ for males and $£$ 3017 for females when depression was present alongside diabetes. When patients had diabetes, CHD and had suffered a stroke these patients' costs were $£ 1541$ for males and $£ 1879$ for females without depression and $£ 3420$ for males and $£ 4072$ for females with depression. Depression increased the associated cost of any comorbidity.

Conclusion The prevalence of depression appears to be more strongly determined by the number of comorbidities rather than the precise nature of the comorbid diagnoses. Additional costs of health care utilisation are considerably higher when depression is associated with single or multiple comorbidities.

\section{PS29 YOUTH EXPOSURE TO ONLINE ALCOHOL ADVERTISING IN THE UK: AN ANALYSIS OF SOCIAL MEDIA WEBSITES}

doi:10.1136/jech-2012-201753.128

'E Winpenny, ${ }^{2} \mathrm{TM}$ Marteau, ${ }^{1} \mathrm{E}$ Nolte. ${ }^{1}$ Health $\&$ Healthcare, RAND Europe, Cambridge, UK; ${ }^{2}$ Behaviour and Health Research Unit, University of Cambridge, Cambridge, UK

Background There is increasing evidence that youth exposure to alcohol marketing is a risk factor for underage drinking. In 2011, online marketing became the largest channel for marketing for the first time, overtaking television. However, there is little understanding of the level of exposure of young people to online alcohol marketing.

Methods We obtained data on the top 3 social media sites in the UK for each month from December 2010 to May 2011, based on unique user figures, by gender and age (6-14, 15-24). We analysed the reach (the proportion of available internet users who used the site in each month) and impressions (the number of individual pages viewed on the site in each month) of the overall top three social media sites, Facebook, YouTube and Twitter in each demographic. Using data from the top 10 TV channels in the UK we identified 5 drinks brands, which had the highest TV advertising exposure to children (4-15) during this 6 month period. During February and March 2012, we examined each of these brands across the 